$$
\begin{aligned}
& \text { تقيم أداء Hydrus-1D في محاكاة التبخر من سطح التربة لفواصل ترطيب مختفة }
\end{aligned}
$$

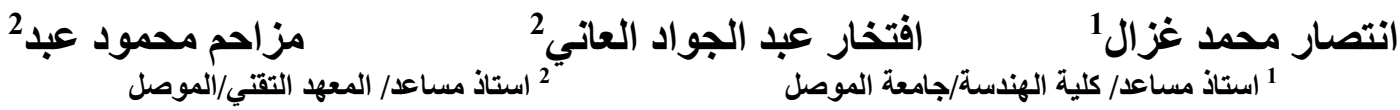

$$
\begin{aligned}
& \text { الملخص }
\end{aligned}
$$

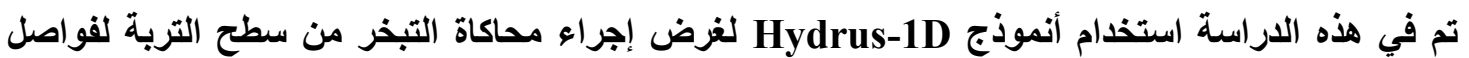

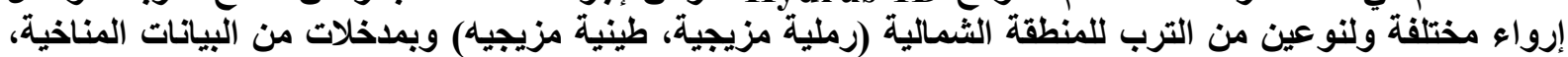

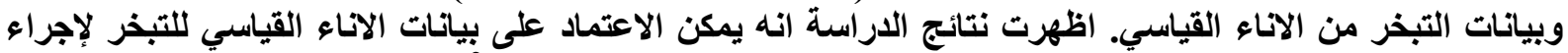

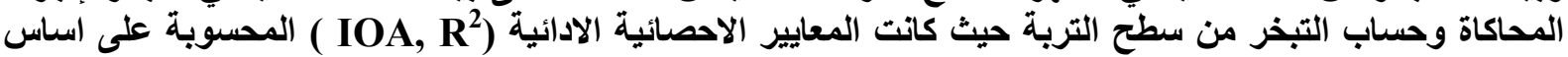

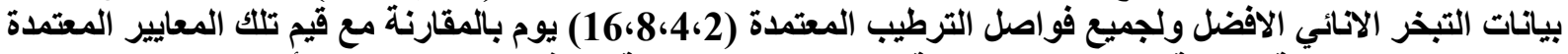

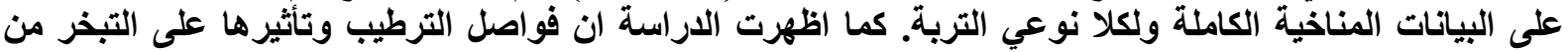

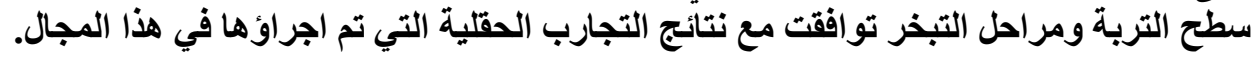

\title{
Evaluating performance of Hydrus-1D model in simulating evaporation from soil surface for different wetting intervals
}

\author{
Entesar M.Ghazal \\ Eftikhar A. Alani \\ Muzahim M. Abi
}

\begin{abstract}
In this study, Hydrus-1D model was used to simulate the evaporation from the soil surface for different wetting intervals for northern region(sandy loam and clay loam) using input for climatic data, and data of class A pan evaporation. The results showed that data of pan evaporation class $A$ can be relied on in simulating and calculation of evaporation from the soil surface, where the standards of statistics (IOA, $\mathbf{R}^{2}$ ) calculated on the basis of evaporation pan class $A$ data are better for the wetting intervals $(2,4,8,16)$ days compared with the values of those standards based on full climate data and both types of soil. The study also showed that the wetting intervals and their impact on evaporation from the soil surface and evaporation stages reasonably agree with the results of field experiments that have been made in this area.
\end{abstract}

Keywords: Hydrus-1D model, soil surface evaporation wetting intervals 
المقدمة

يعد الماء عاملا رئيسأ ومهما في الزراعة الإروائية، ولكن المصادر المائية المائية محدودة، وأن للوحدة المائية كلفتها

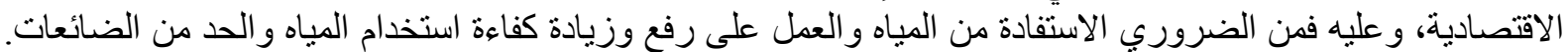

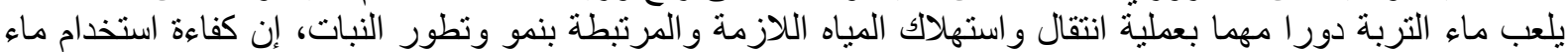

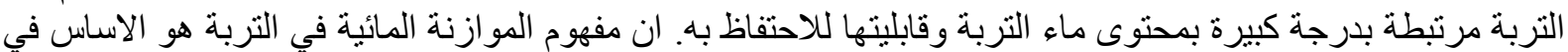

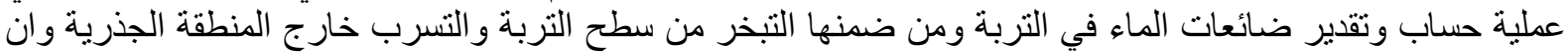

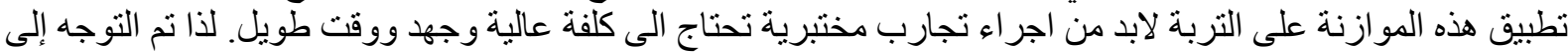

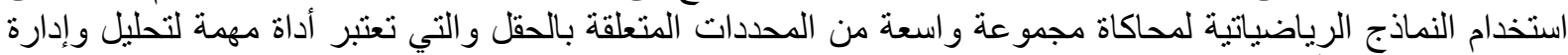

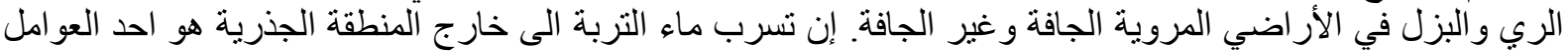

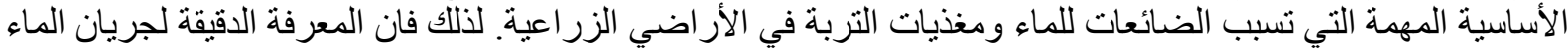

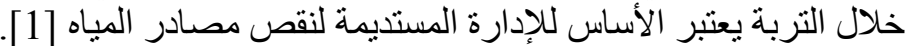

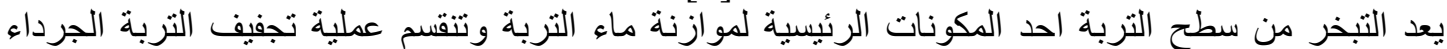

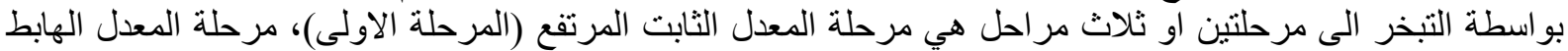

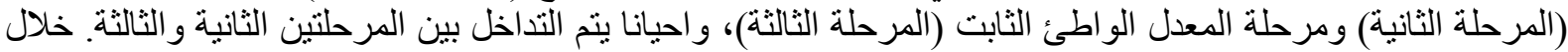

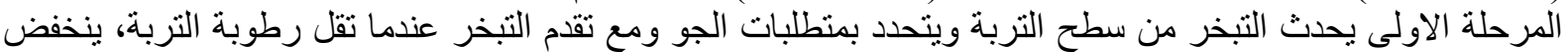

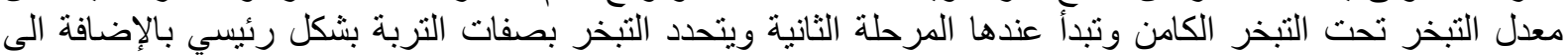

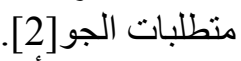

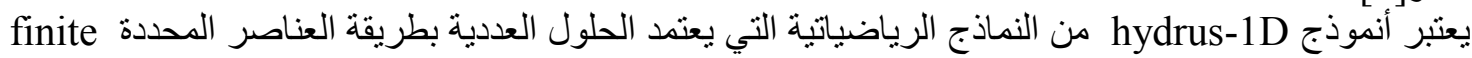

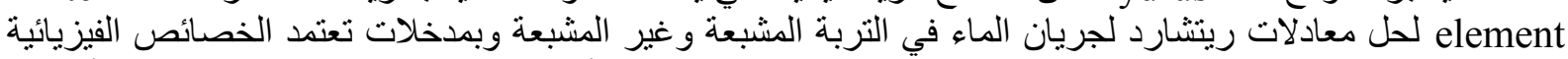

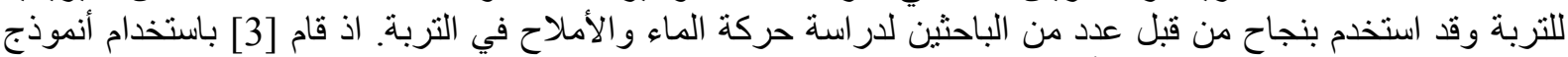

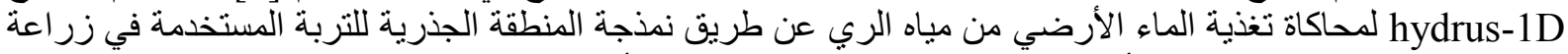

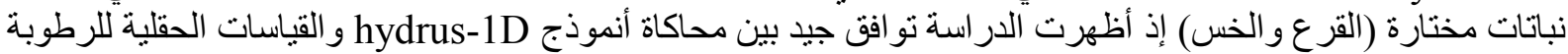
في منطقة مختارة في اسبانيا .

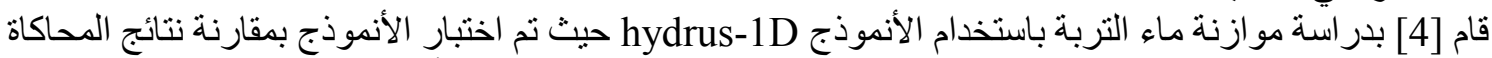

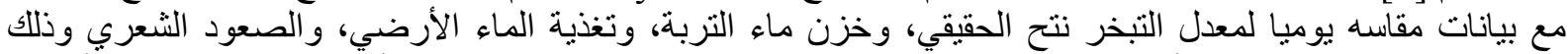

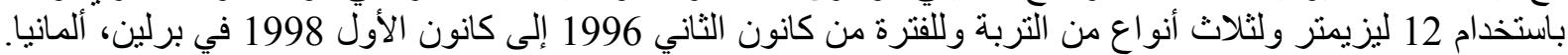

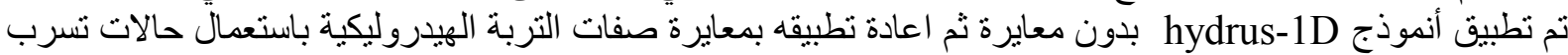

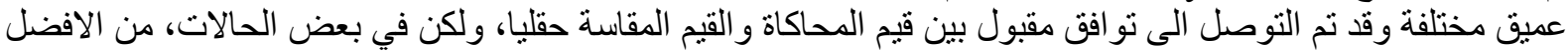

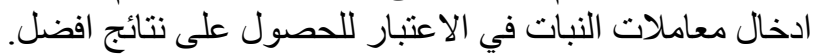

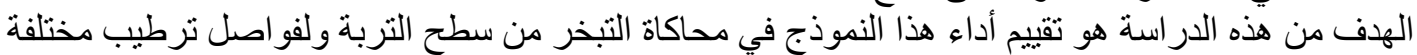

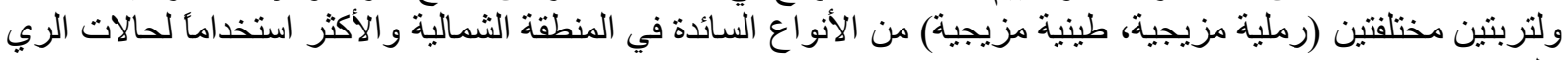
بالرش.

الاسكاس النظري

Hydrus-1D

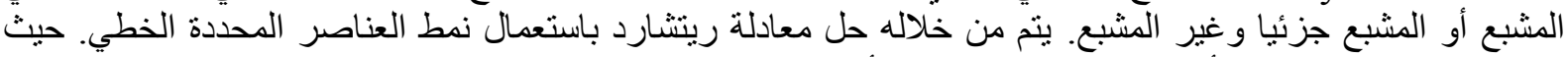

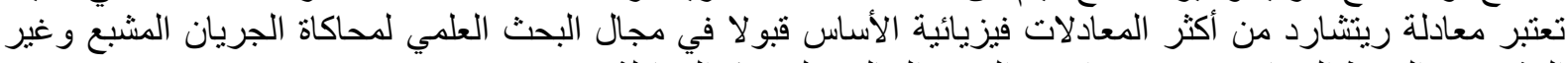

$$
\frac{\partial \boldsymbol{\theta}}{\partial \boldsymbol{t}}=\frac{\partial}{\partial \boldsymbol{z}}\left[(\boldsymbol{k}(\boldsymbol{h}))\left(\frac{\partial \boldsymbol{h}}{\partial \boldsymbol{z}}+1\right)\right]-\boldsymbol{S}
$$

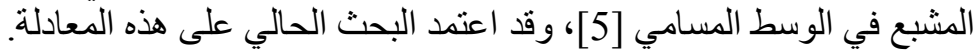

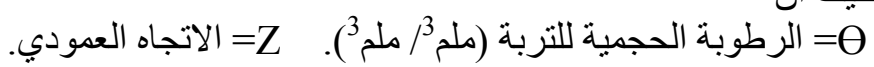

$$
\text { K(h) }
$$

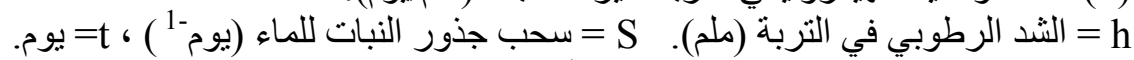

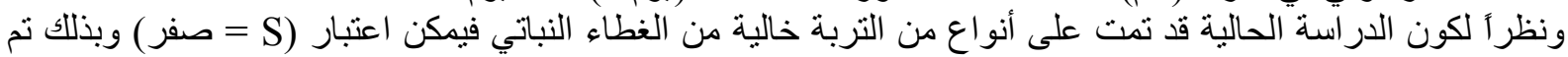
تكييف صيغة معادلة ريتشارد كما يلي : 


$$
\frac{\partial \boldsymbol{\theta}}{\partial \boldsymbol{t}}=\frac{\partial}{\partial \boldsymbol{z}}\left[(\boldsymbol{k}(\boldsymbol{h}))\left(\frac{\partial \boldsymbol{h}}{\partial \boldsymbol{z}}+1\right)\right]
$$

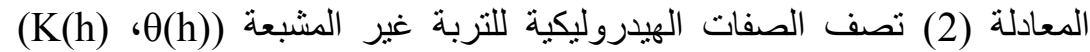

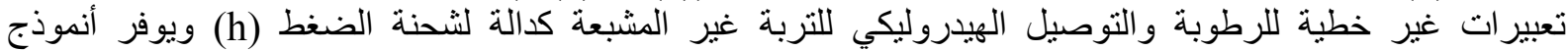
إمكانية استخدام نماذج تحليلية لوصف هذه الصفات للتربة. وقد تم وصف الصفية الصفات الهيدروليكية للتربة حسب أنموذج van Genuchten -Mualem, وكما يلي:

$\theta(h)=\left\{\theta r+\frac{1}{\left[1+|\alpha h|^{n}\right]^{m}} \quad \mathrm{~h}<0\right.$

$K(h)=K S * S_{e}^{1} *\left[1-\left(1-S_{e}^{1 / m}\right)^{m}\right]^{2}$

$S_{e}=\frac{\theta-\theta_{r}}{\theta_{s}-\theta_{r}}$

Or

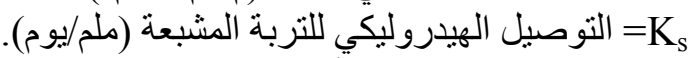

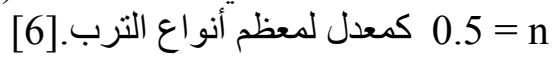

ويمكن تخمين متغيرات انموذج

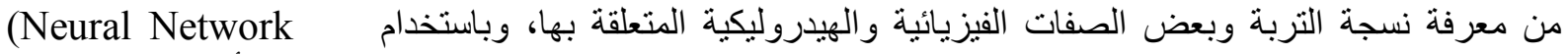

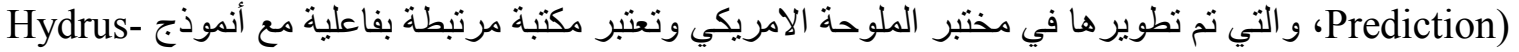

ولحل معادلة ريتشارد لابد من معرفة التوزيع الأولي للظروف المحيطية في مجال الجريان.

$h(z, t)=h_{i}(Z) \quad$ at $\quad t=0$

$h(z, t)=h_{L}(t) \quad$ at $\quad z=L$

ويضاف إليها الحدود المحيطية التالية:

$h(0, t)=h_{A} \quad$ for $\quad h<h_{A}$

$h(0, t)=h_{S} \quad$ for $\quad h>h_{S}$

إذ = أقل شحنة ضغط مسموح بها فوق سطح التربة (ملم) تحت الظروف الجوية المعتمدة. ويتم إيجاد قيمتها من تحديد

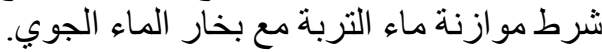

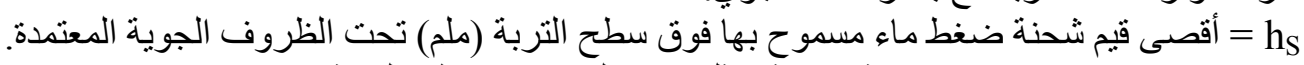
وقيمتها تساوي صفر او قيمة موجبة تمثل عمق الماء فوق سطح التربة فلته 


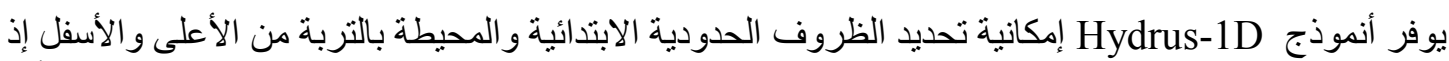

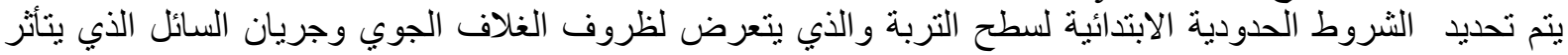

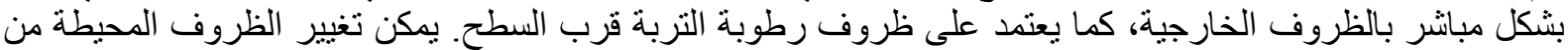

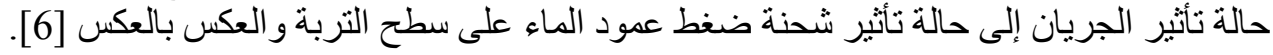

\section{المواد وطرق العمل}

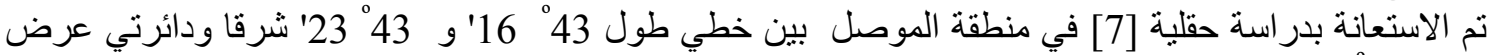

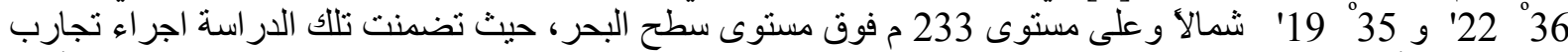

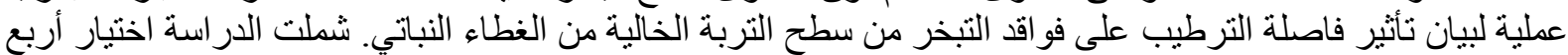

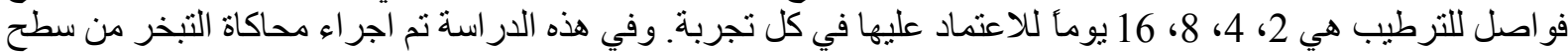

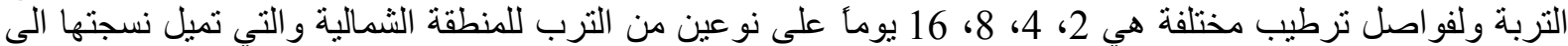

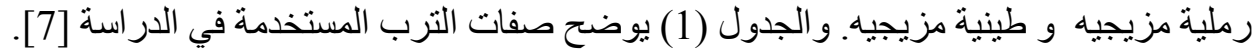

الجدول (1) صفات الترب المستخدمة في الدراسة.

\begin{tabular}{|c|c|c|c|c|c|}
\hline الرطوبة الابتدائية & الرطوبة عند السعة الحقلية & 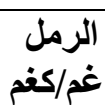 & غ الغرين & غم/كغين & نوع التربية \\
\hline 0.28 & 0.36 & 594 & 282 & 124 & رملية مزيجية \\
\hline 0.33 & 0.41 & 384 & 274 & 342 & طينية مزيجية \\
\hline
\end{tabular}

\section{تطبيق الأنموذج}

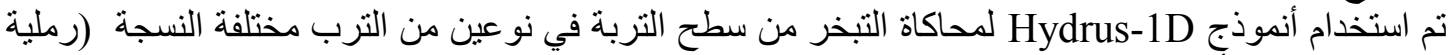

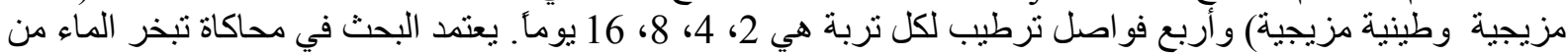

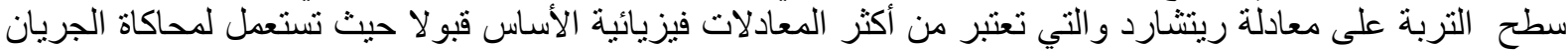

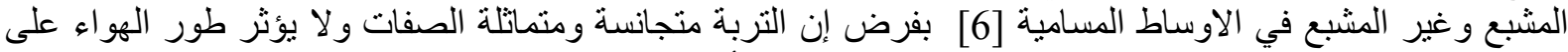

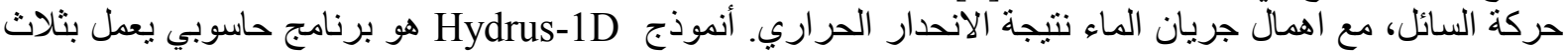

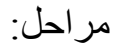

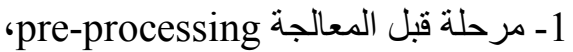

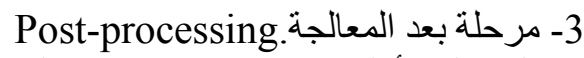

2-2 مرحلة الحسابات (calculation)

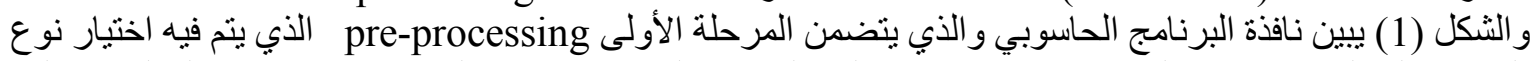

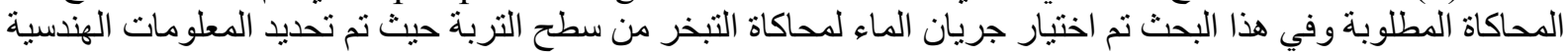
باعتبار مقد التربة يتكون من طبقة واحدة منجانسة بعمق ( 800 ملم) كما في الثكل (2).

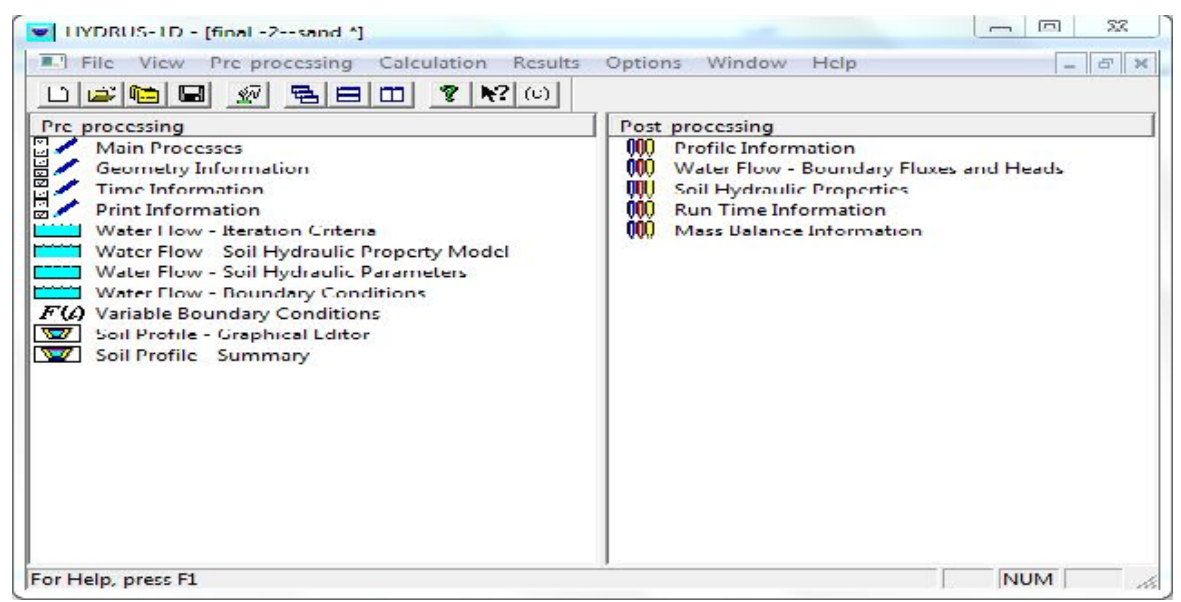

Hydrurs-1D الثكل (1) يوضح نافذة فتح برنامج 


\section{غزال: تقييم أداء Hydrus-1D في محاكاة التبخر من سطح التربة لفواصل ترطيب مختلفة}

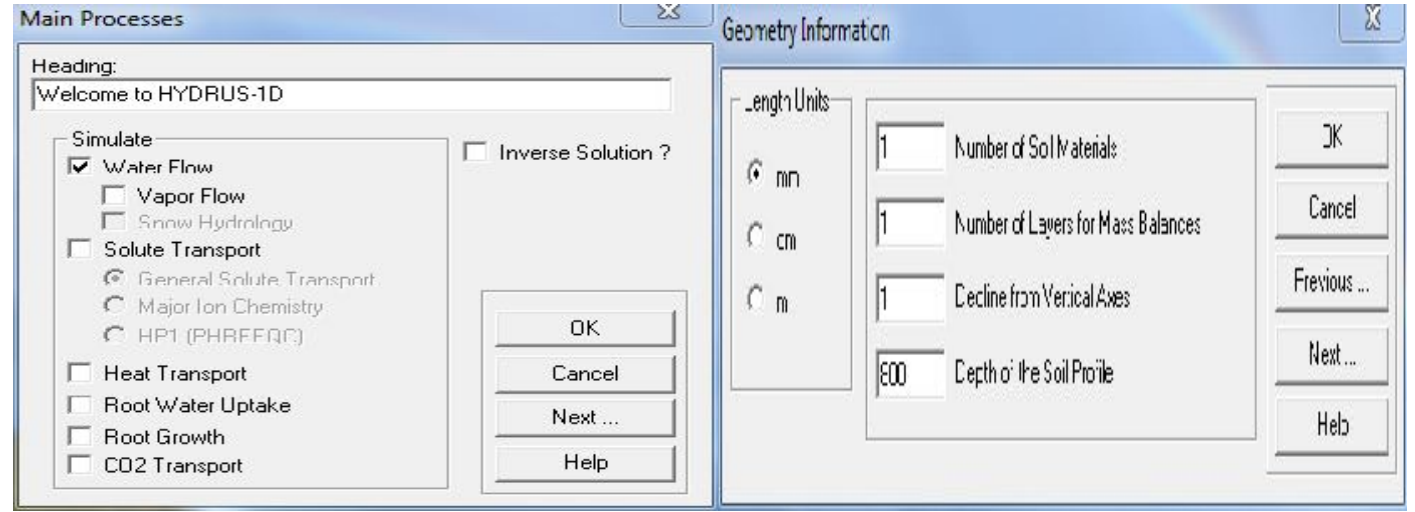

الثكل(2) اختيار نوع المحاكاة و المعلومات الهندية لمقد التربة.

و اعتمادأ على فواصل الترطيب قيد الدر اسة (2، 4، 8، 16 يومأ) فقد تم اختيار الزمن الاولي للمحاكاة (صفر يوم ) و الزمن

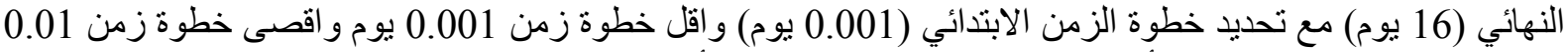

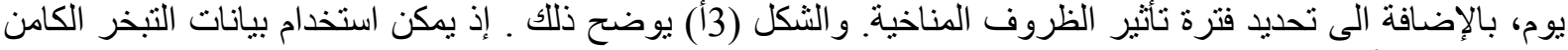

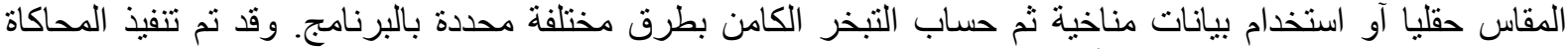

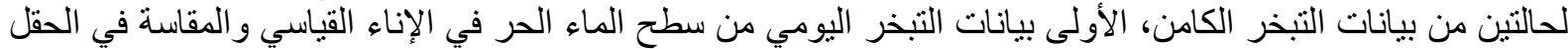

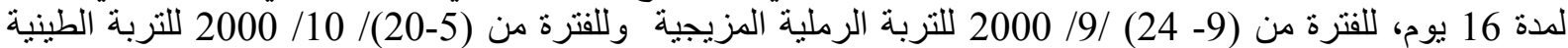

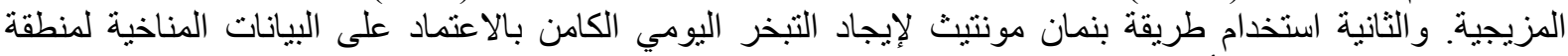
الموصل ولنفس الفترة التي أجريت فيها التجارب، كما موضح في النئة الثكل (3 ب).

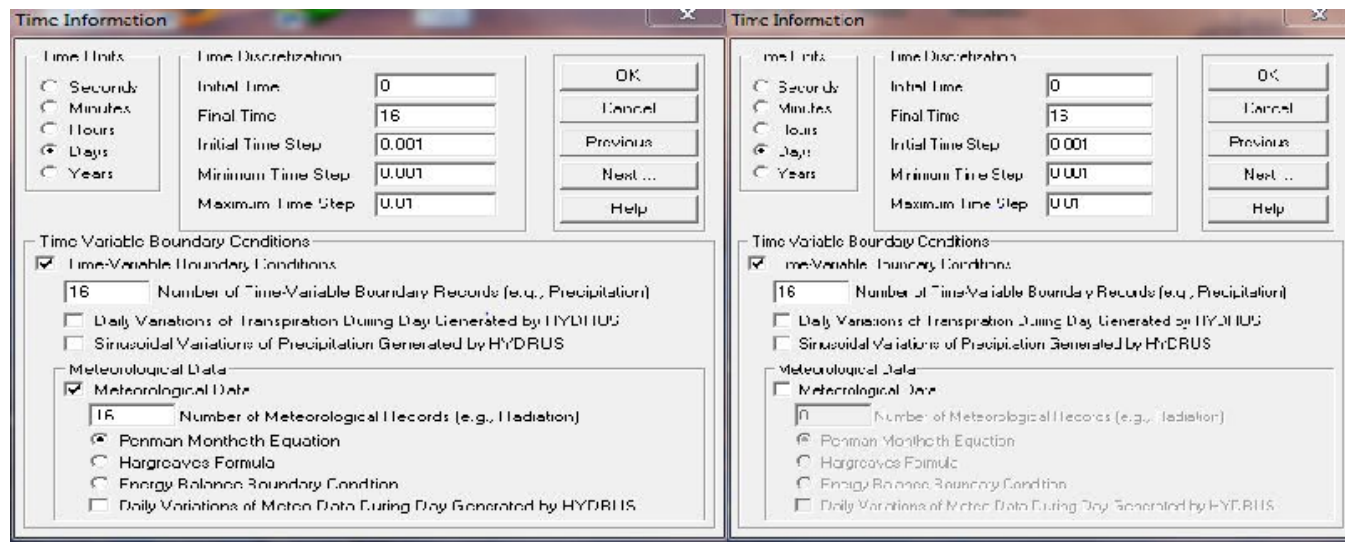

(ب)

الثكل (3) توضيح لبيانات الزمن وفترة تأثنير الظروف المناخية.

(l)

لغرض تحديد أفضل البيانات لإجراء المحاكاة لابد من تحديد معايير التكرار التي يتم بواسطتها حل معادلة

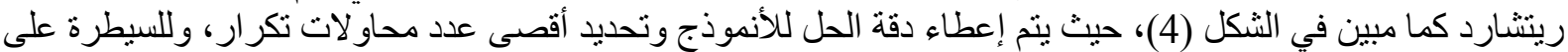

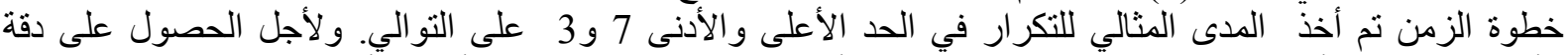

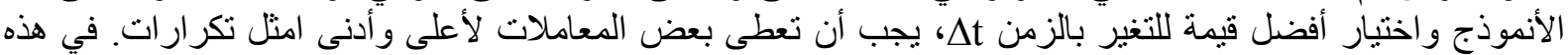

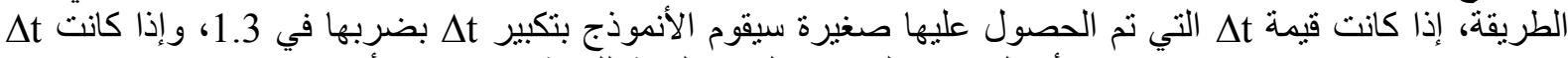

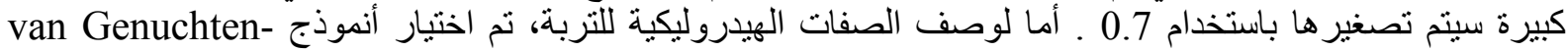
Mualem مع اعتبار ظاهرة التخلف ابتداء| بمنحنى الترطيب كما في الثكل (5). 


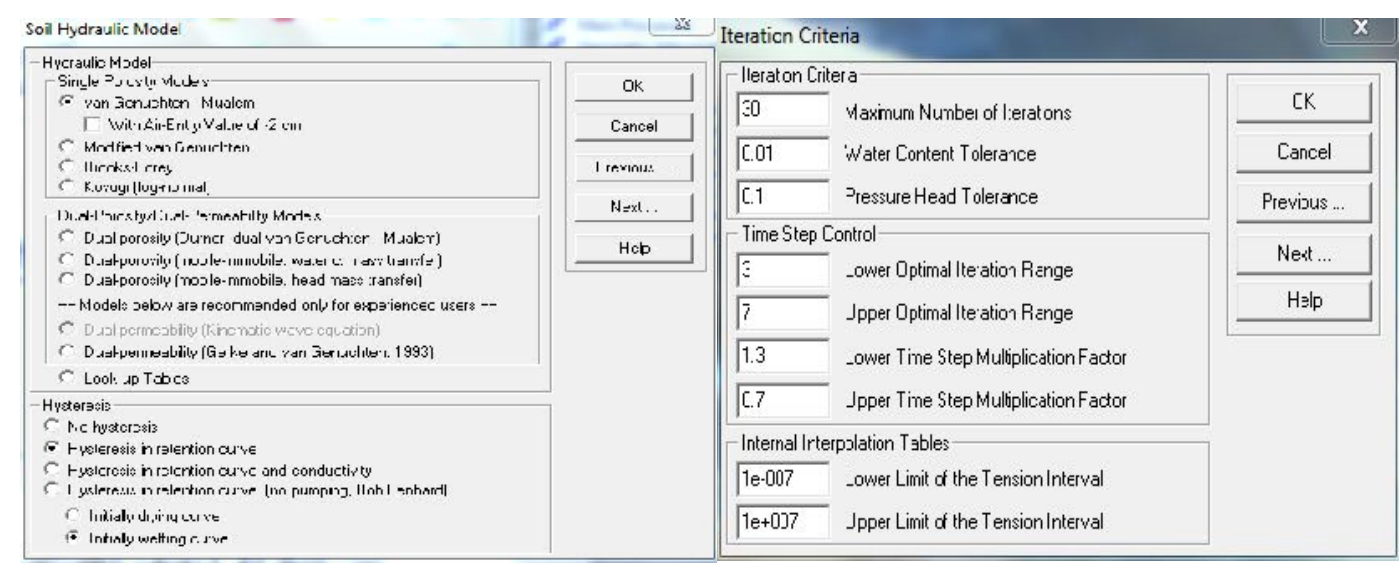

الثكل (5) وصف الصفات الهيدروليكية للتربة الشكل(4) معايير التكرار المستخدمة .

تم تقدير متغيرات أنموذج

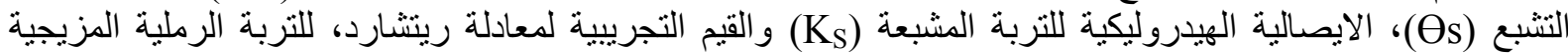

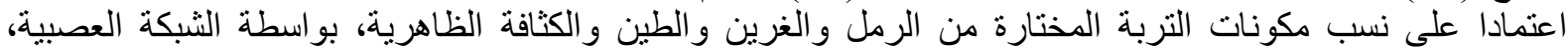

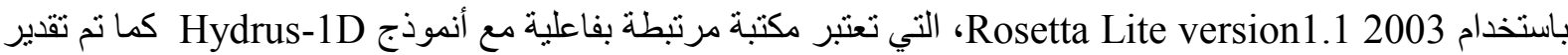

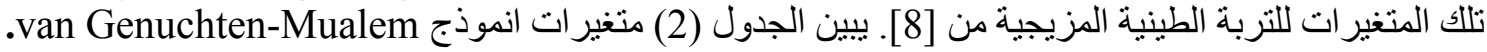
الجدول (2) متغير ات انموذج van Genuchten-Mualem

\begin{tabular}{|c|c|c|c|c|c|c|}
\hline ملم/يوم Ks & I & n & 1 1/ملم & $\boldsymbol{\theta s}$ & Or & التربة \\
\hline 428 & 0.5 & 1.452 & 0.0021 & 0.4 & 0.047 & رملية مزيجية \\
\hline 105 & 0.5 & 1.37 & 0.003 & 0.47 & 0.129 & طينية مزيجية \\
\hline
\end{tabular}

الظروف الحدودية المحيطة

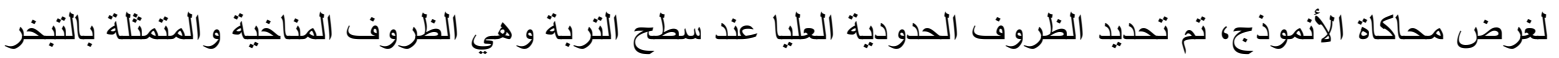

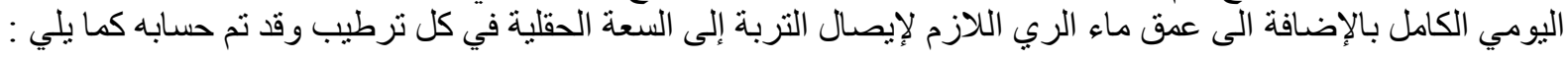
$I=(\theta f c-\theta i) Z$

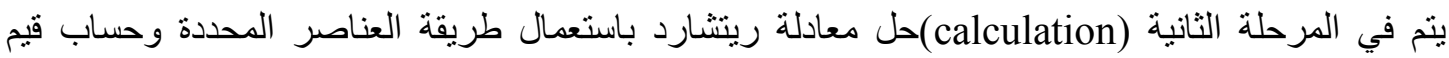

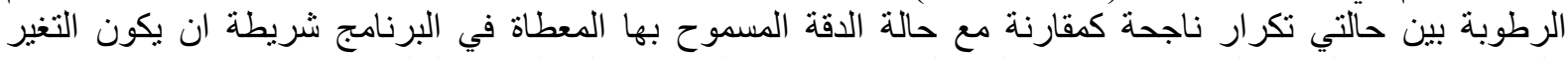

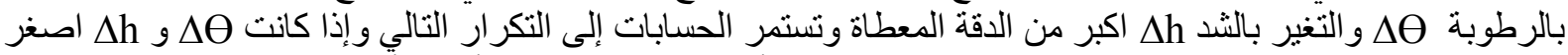

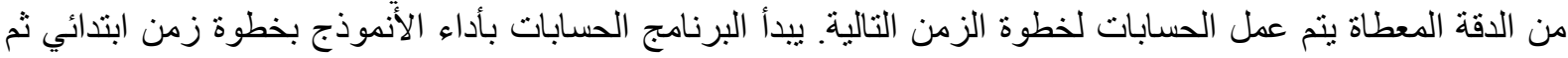


مقارنة القيم المستتتجة في التكرار مع الدقة المعطاة، وفي النهاية يتم ترتيب القيم المعدلة حسب أعلى وأدنى خطوة زمن

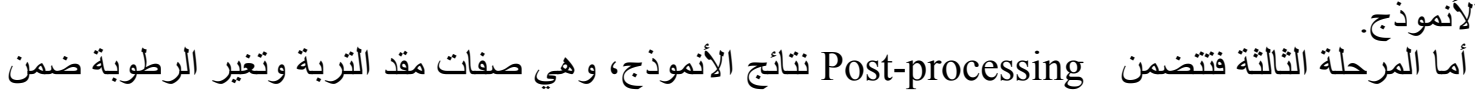
مقد التربة مع الزمن، الصفات الهيدروليكية للتربة، جريان الماء، و الموازنة المائية في التربة و التي تظهر على شكل جداول

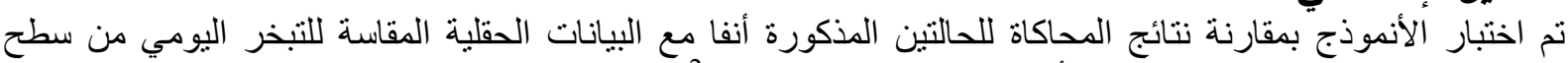

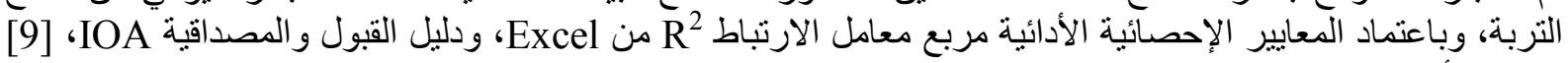

$$
\begin{gathered}
R^{2}=\left\{\frac{\sum_{i=1}^{N}\left(E_{f}-E_{m f}\right)\left(E_{h}-E_{m h}\right)}{\sqrt{\sum_{i=1}^{N}\left(E_{f}-E_{m f}\right)^{2}\left(E_{h}-E_{m h}\right)^{2}}}\right\}^{2} \\
I O A=1-\frac{\sum_{i=1}^{N}\left(E_{f}-E_{h}\right)^{2}}{\sum_{i=1}^{N}\left(a b s\left(E_{f}-E_{m f}\right)+a b s\left(E_{h}-E_{m h}\right)\right)^{2}}
\end{gathered}
$$

Ef E = E E = Emf

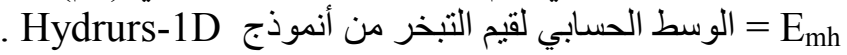
N = عدد البيانات.

\section{النتائج والمناقشة}

تم استخدام بيانات التبخر الانائي المقاس حقليا بو اسطة اناء التبخر(A) و البيانات المناخية لمنطقة الموصل للفترة

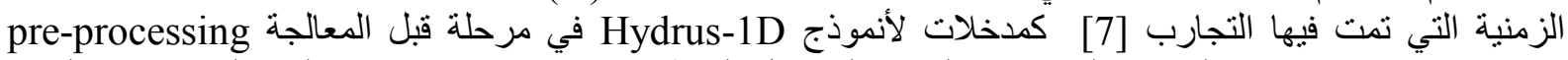

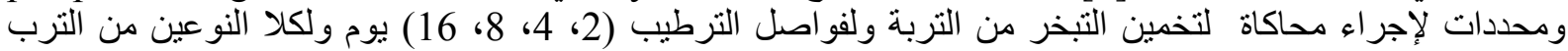

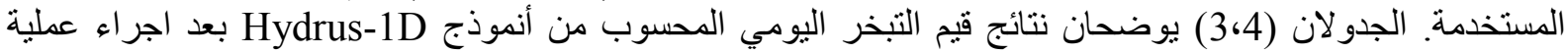

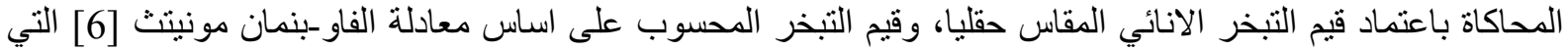

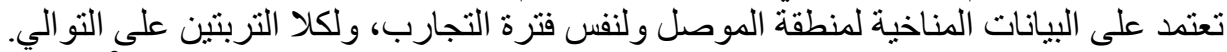

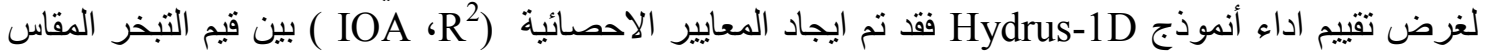

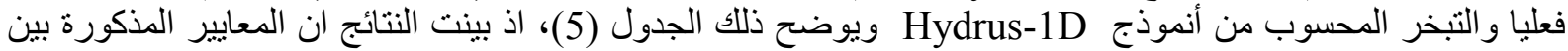

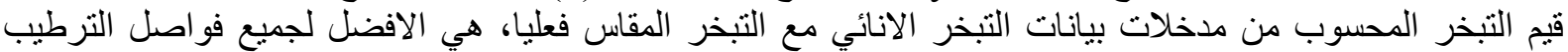

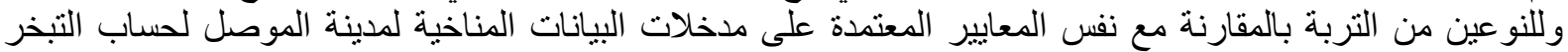

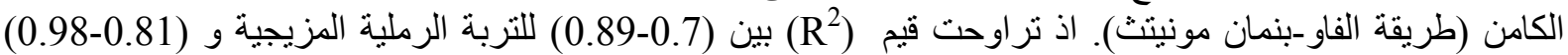

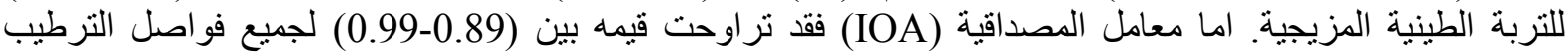

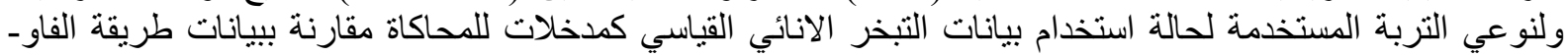

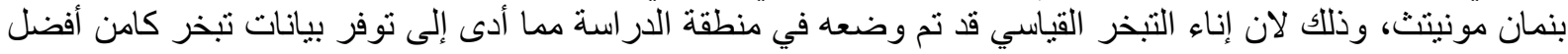

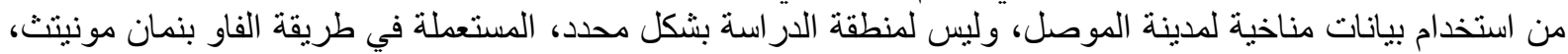

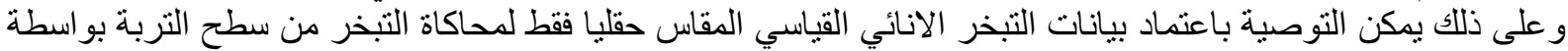

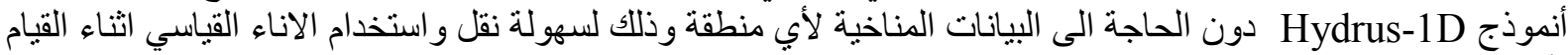


لبيان معنوية الفروقات تم اجراء بaired t-test بين قيم التبخر اليومي المحسوب باستخدام أنموذج المبات

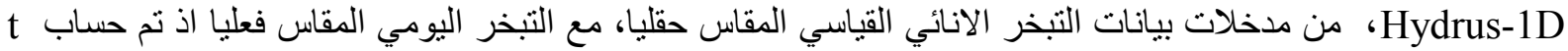

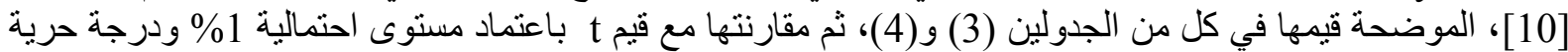

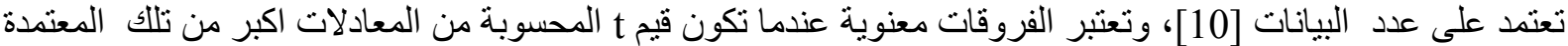

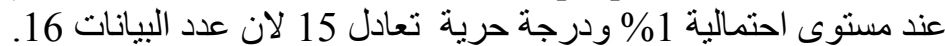
لتوضيح مر احل التبخر وتحديد اوقات حدوثها لكل حالة ترطيب 2،

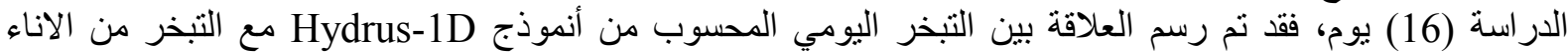

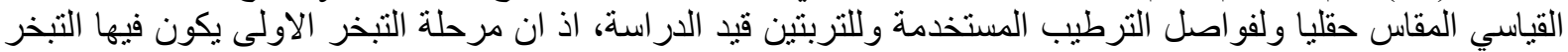

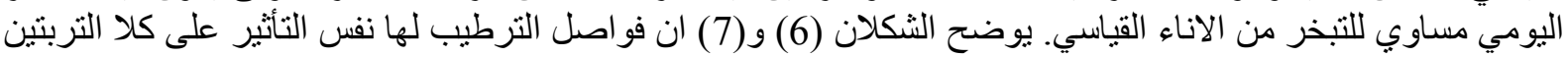

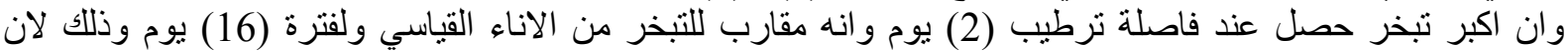

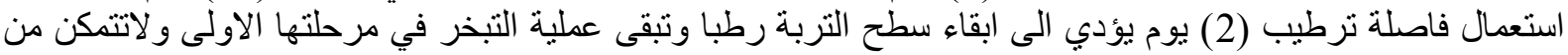

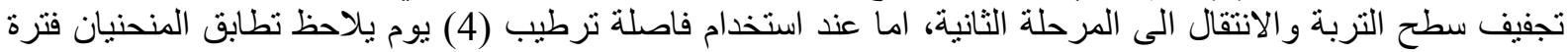

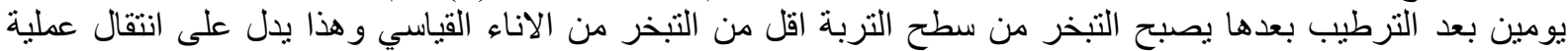

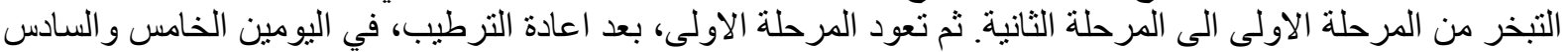

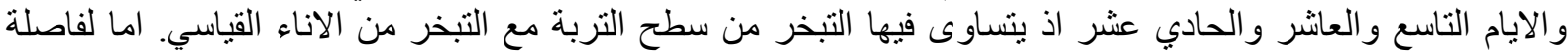

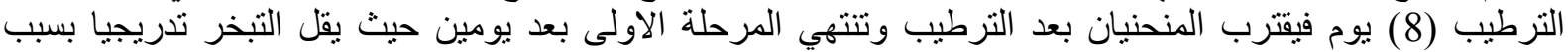

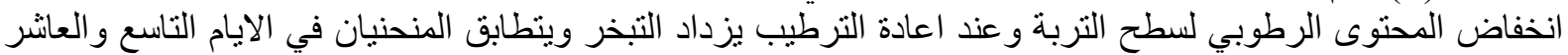

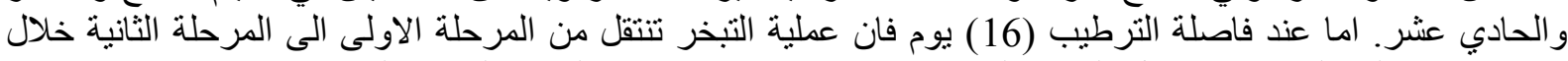

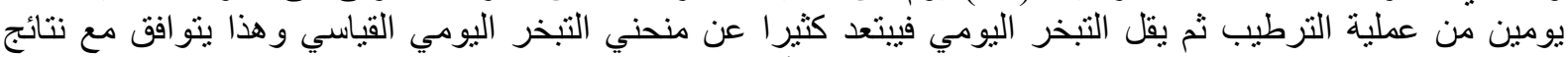

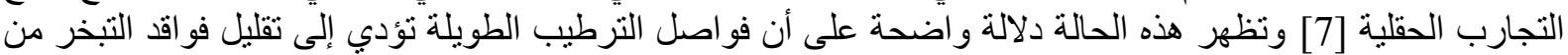
سطح التربة.

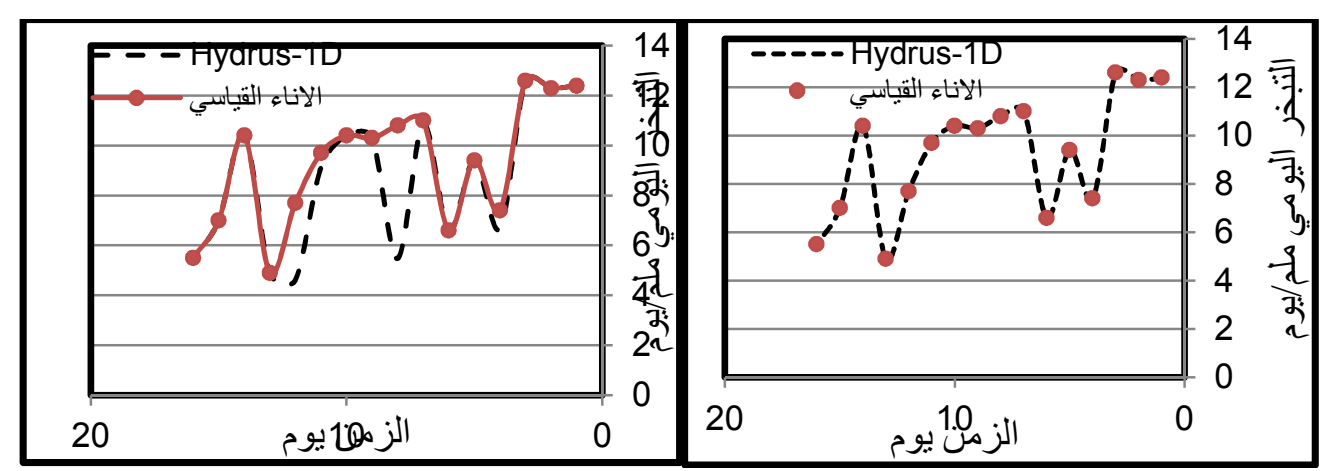

فاصلة ترطيب 4 يوم

فاصلة ترطيب 2 يوم

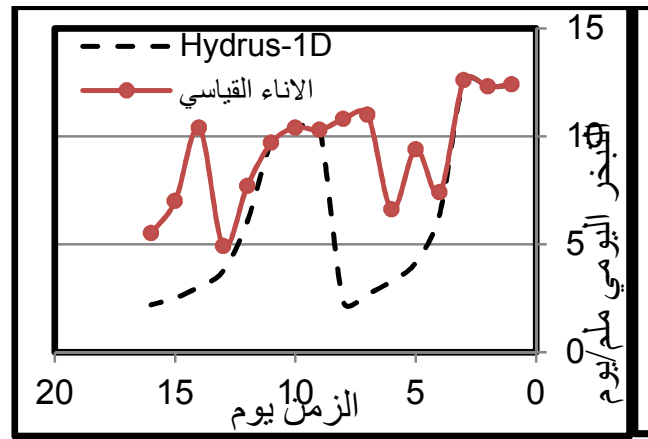

فاصلة ترطيب 16 يوم

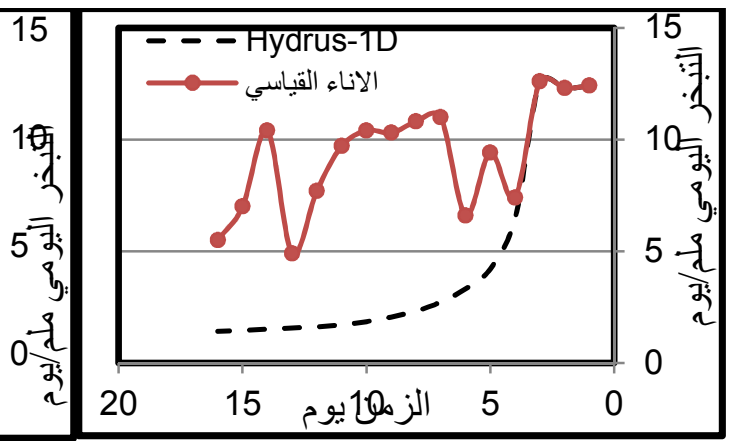

فاصلة ترطيب 8 يوم

الثكل(6) مقارنة تغير التبخر اليومي من سطح التربة مع التبخر اليومي من الاناء القياسي مع الزمن لفو اصل الترطيب ترطي المختلفة للتربة الرملية المزيجية. 
No. 2

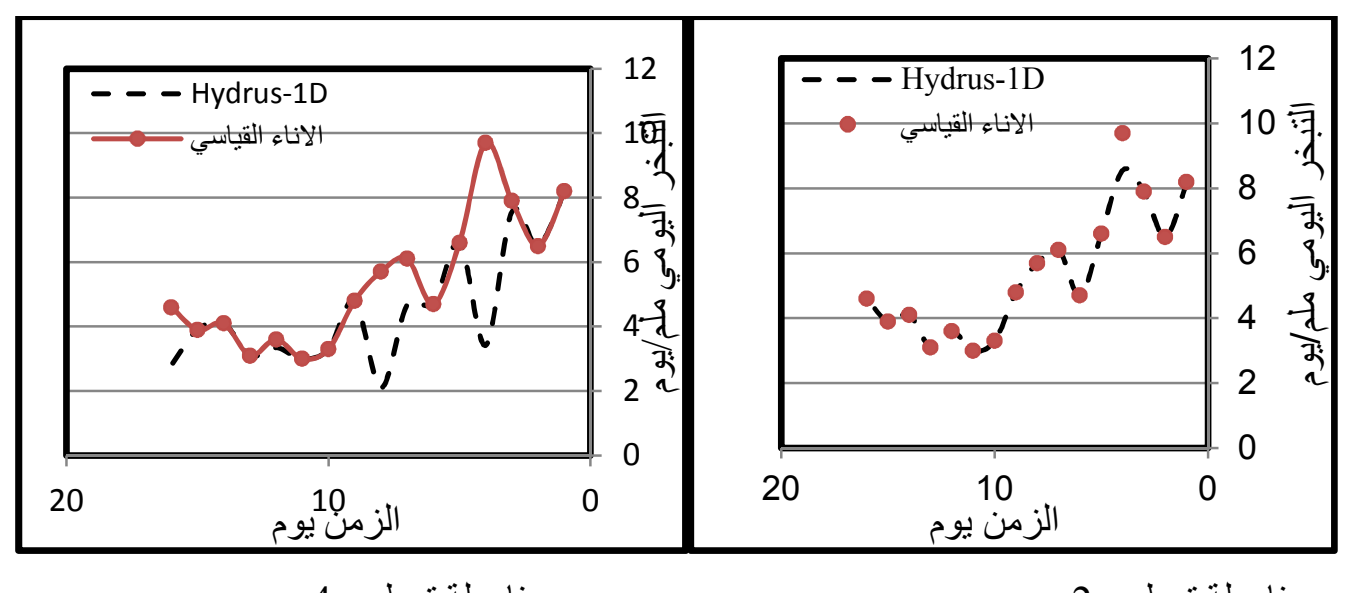

فاصلة ترطيب 4 يوم فاصلة ترطيب 2 يوم
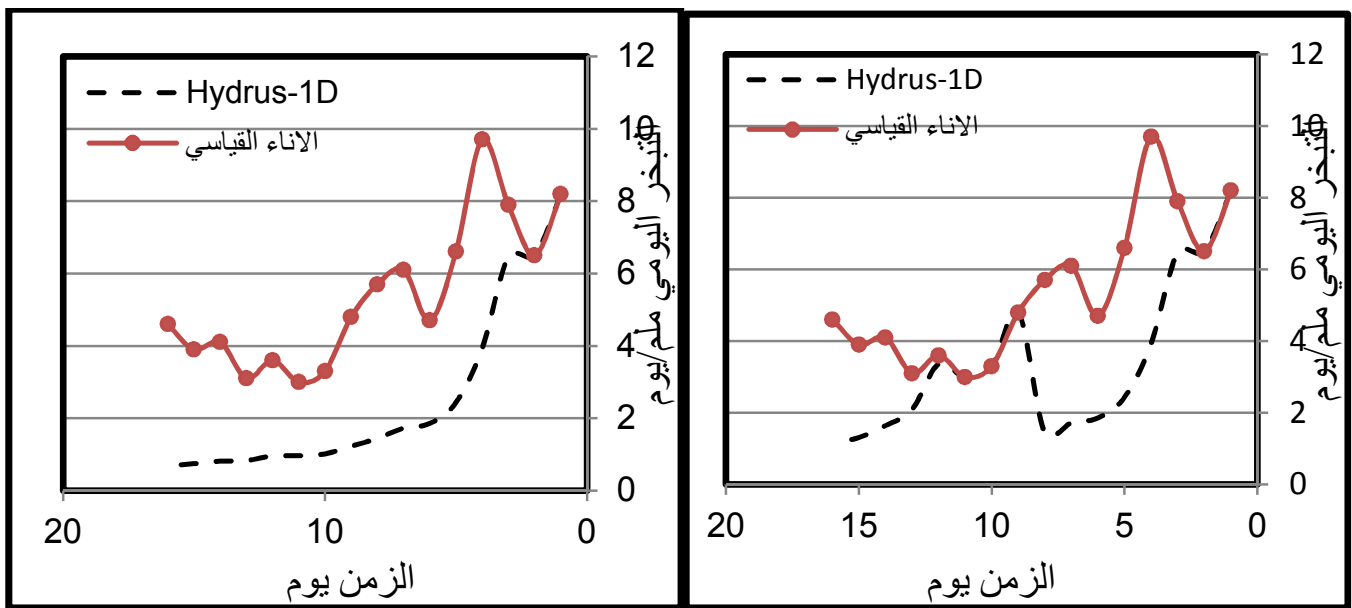

فاصلة ترطيب 16 يوم

$$
\text { فاصلة ترطيب } 8 \text { يوم }
$$

الثكل(7) مقارنة تغير التبخر اليومي من سطح التربة مع التبخر اليومي من الاناء القياسي مع الزمن لفواصل الترطيب المختلفة للتربة الطينية المزيجية. 
غزال: تقييم أداء Hydrus-1D في محاكاة التبخر من سطح التربة لفواصل ترطيب مختلفة

7.

特司高

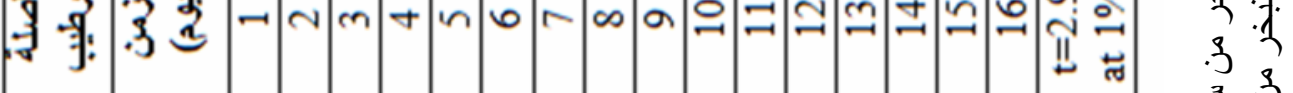

$3 \cdot 3$

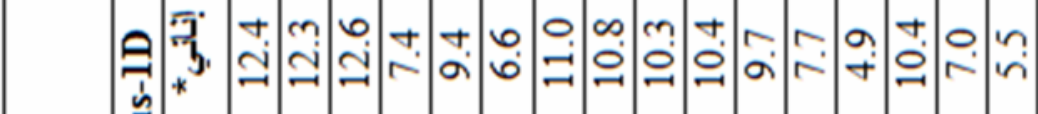

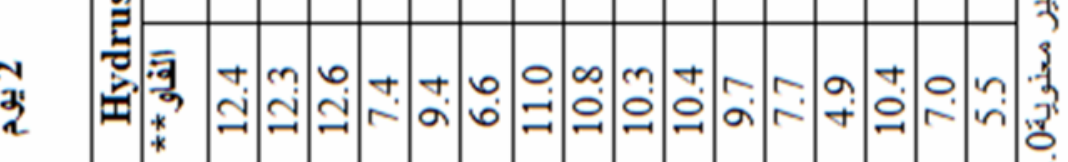

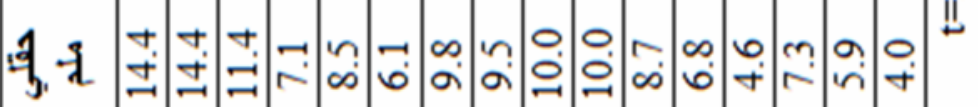

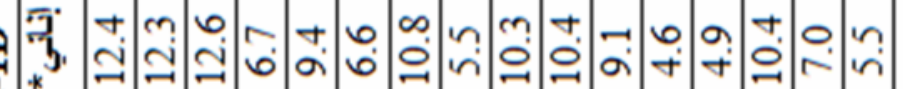

-

司的

.

$3: \frac{1}{3}$

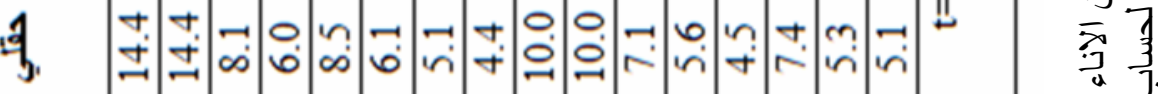

司: 司.

J.

ق

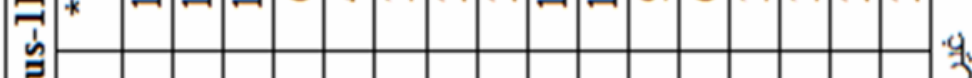

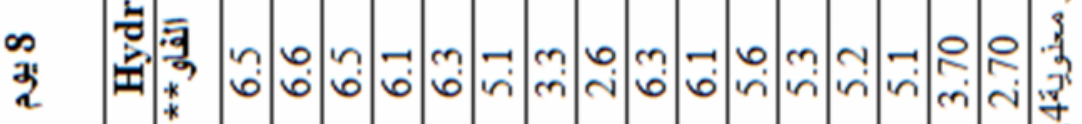

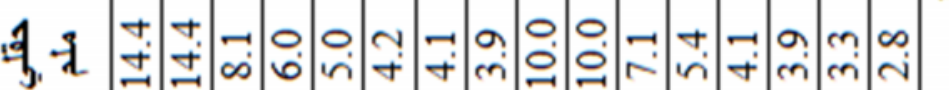

京可

. 3

के -

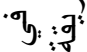

高奇

.

3

:

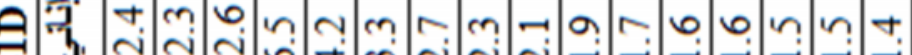

气 * $=-10$ m

7.

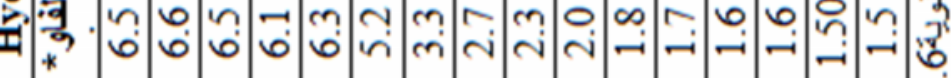

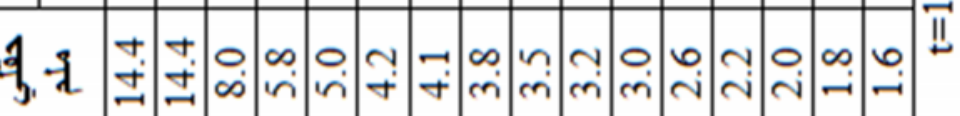


No. 2

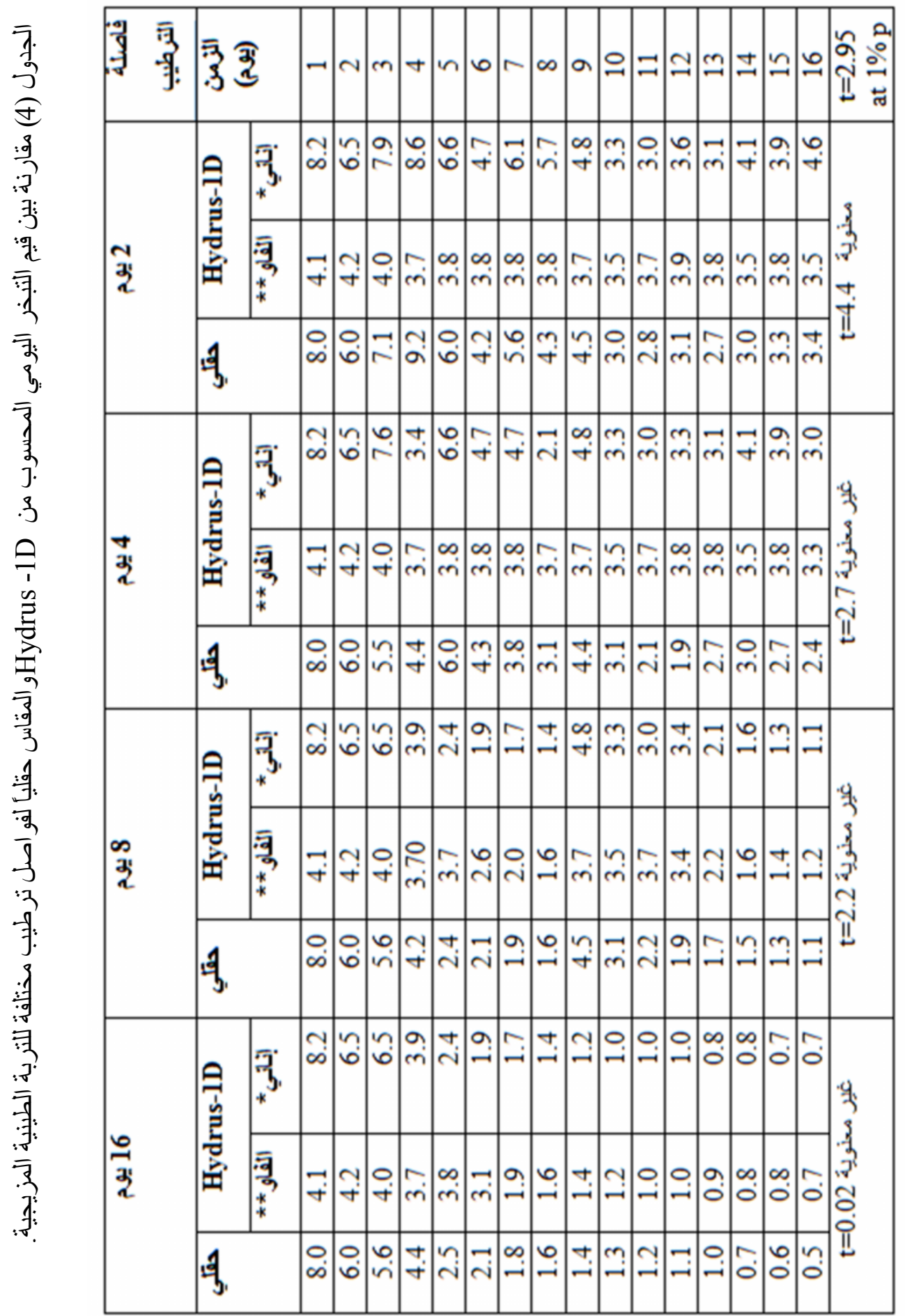


الجدول (5) نتائج المعايير الأدائية والإحصائية للتبخر من البيانات الحقلية.

\begin{tabular}{|c|c|c|c|c|c|}
\hline \multicolumn{2}{|c|}{ طنمان مونيتث } & \multicolumn{2}{|c|}{ التبخر من الإناء } & \multirow{2}{*}{ فاصلة } & \multirow[t]{2}{*}{ التربة } \\
\hline IOA & $\mathrm{R}^{2}$ & IOA & $\mathrm{R}^{2}$ & & \\
\hline 0.96 & 0.89 & 0.96 & 0.89 & 2 & رملية \\
\hline 0.55 & 0.45 & 0.89 & 0.7 & 4 & \\
\hline 0.67 & 0.56 & 0.96 & 0.85 & 8 & \\
\hline 0.74 & 0.64 & 0.966 & 0.87 & 16 & \\
\hline-0.01 & 0.30 & 0.97 & 0.95 & 2 & طينية \\
\hline 0.3 & 0.42 & 0.92 & 0.81 & 4 & \\
\hline 0.78 & 0.59 & 0.98 & 0.95 & 8 & \\
\hline 0.87 & 0.77 & 0.99 & 0.98 & 16 & \\
\hline
\end{tabular}

الاستنتاجات والتوصيات

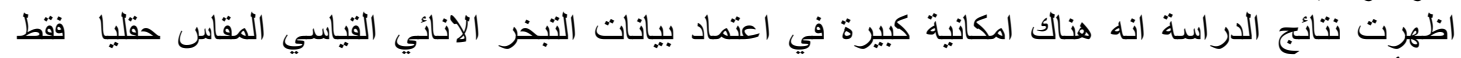

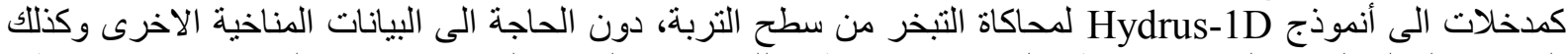

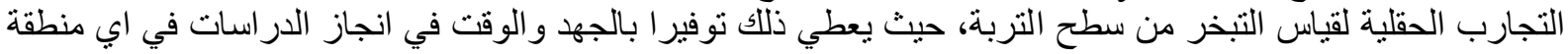

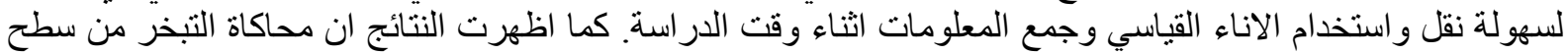

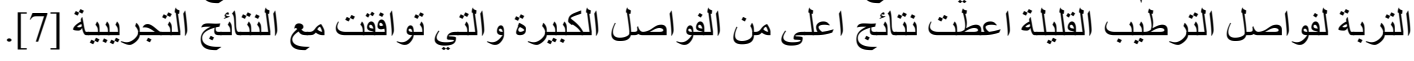

1-Skaggs, T.H, Van Genuchten, M.TH, Shouse, P.J and Poss, J.A.(2006). " acroscopic approaches to root water uptake as a function of water and salinity stress." Agricultural water management . 86, 140-149.

2- Jiangbo Han and Zhifang Zhou. (2013). " Dynamics of Soil Water Evaporation during Soil Drying: Laboratory Experiment and Numerical Analysis". The Scientific World Journal Volume 2013 (2013), Article ID 240280, 10

pages.http://dx.doi.org/10.1155/2013/240280.

3- Jimenez-Martinez, J , Skaggs, T.H, Van Genuchten, M.TH. and Candela, L .(2009)

."A root zone modeling approach to estimating groundwater recharge from irrigated areas". Journal of hydrology 367, 138-149.

4- Wegehenkel, M.(2009). "The estimation of soil water fluxes using lysimeter data". EGU general assembly 2009, held 19-24 April, 2009 in vienna.

5- Soylu, M.E., Estanbulluoglu, E., Lenters, J.D., and Wang, T.(2011)." Quantifying the impact of groundwater depth on evapotranspiration in a semi-arid grassland region". Hydrol. earth syst. sci., 15, 787-806.

6- Simunek, J. ,Sejna, M., Saito, H., Sakai, M and van Genuchten, M. Th.(2009)." The HYDRUS-1D Software Package for Simulating the One-Dimensional Movement of Water, 
Heat, and Multiple Solutes in Variably-Saturated Media. Version 4.08. Department of environmental Sciences University of California Riverside , USA, 296 pp.

$$
\begin{aligned}
& \text { 7- اسوفي، سنان عدنان سليمان يوسف. (2001)." تأثثر فاصلة الإرواء في فو اقد التبخر لمياه الري". } \\
& \text { رسالة ماجستير ، جامعة الموصل، كلية الهندسة . }
\end{aligned}
$$

8- Leij, Feike J., Alves, William J., Van Gentencten, Martinus Th. and Williams ,Joseph R.(1966). "The UNSODA Unsaturated Soil Hydraulic Database, User's Manual Version 1.0, EPA/600/R-96/095. August1996.

9- Helmers, Qi, Z., M, and Singh, R. 2006. "Evaluating a drainage model using soil hydraulic parameters derived from various methods". ASAE Meeting Paper No. 062318. St. Joseph, Mich. :ASAE.

10-Ractliffe, J.F. (1972)."Element of Mathematical Statistics. Oxford Mathematical Handbook. Oxford University Press.Pp224.

$$
\text { تم اجراء البحث في كلية ألهندة = جامعة ألموصل }
$$

